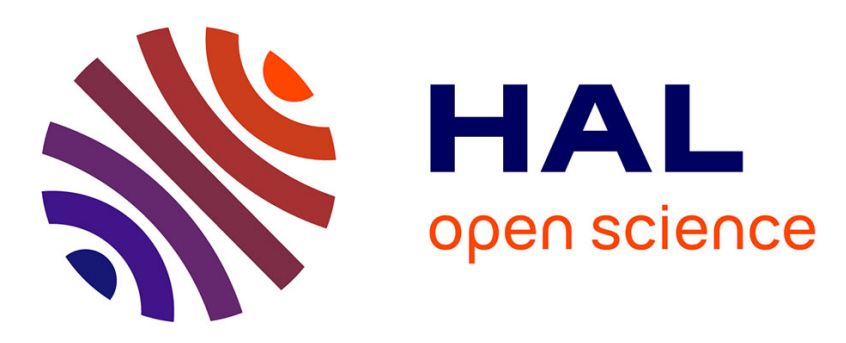

\title{
Singularity Analysis of the H4 Robot using Grassmann-Cayley Algebra
}

Semaan Amine, Stéphane Caro, Philippe Wenger, Daniel Kanaan

\section{To cite this version:}

Semaan Amine, Stéphane Caro, Philippe Wenger, Daniel Kanaan. Singularity Analysis of the H4 Robot using Grassmann-Cayley Algebra. Robotica, 2011, pp.1-10. 10.1017/S0263574711001330 . hal-00642230

\section{HAL Id: hal-00642230 \\ https://hal.science/hal-00642230}

Submitted on 17 Nov 2011

HAL is a multi-disciplinary open access archive for the deposit and dissemination of scientific research documents, whether they are published or not. The documents may come from teaching and research institutions in France or abroad, or from public or private research centers.
L'archive ouverte pluridisciplinaire HAL, est destinée au dépôt et à la diffusion de documents scientifiques de niveau recherche, publiés ou non, émanant des établissements d'enseignement et de recherche français ou étrangers, des laboratoires publics ou privés. 


\title{
Singularity Analysis of the H4 Robot using Grassmann-Cayley Algebra
}

\author{
Semaan Amine, Stéphane Caro, Philippe Wenger and Daniel Kanaan \\ Institut de Recherche en Communications et Cybernétique de Nantes (IRCCyN), \\ 1, rue de la Noë 44321 Nantes, France. \\ E-mail: \{semaan.amine, stephane.caro, philippe.wenger, daniel.kanaan\}@irccyn.ec-nantes.fr, \\ Tel : +332403769 68, fax: +33240376930.
}

\begin{abstract}
This paper extends a recently proposed singularity analysis method to lower-mobility parallel manipulators having an articulated nacelle. Using screw theory, a twist graph is introduced in order to simplify the constraint analysis of such manipulators. Then, a wrench graph is obtained in order to represent some points at infinity on the Plücker lines of the Jacobian matrix. Using GrassmannCayley algebra, the rank deficiency of the Jacobian matrix amounts to the vanishing condition of the superbracket. Accordingly, the parallel singularities are expressed in three different forms involving superbrackets, meet and join operators, and vector cross and dot products, respectively. The approach is explained through the singularity analysis of the $\mathrm{H} 4$ robot. All the singularity conditions of this robot are enumerated and the motions associated with these singularities are characterized.
\end{abstract}

Keywords: Grassmann-Cayley algebra, parallel singularity, screw theory, articulated nacelle, projective space, superbracket.

\section{Introduction}

Parallel singularities are critical configurations in which a Parallel Manipulator (PM) loses its inherent stiffness and the motion of its end-effector becomes uncontrollable. Generally, such configurations are related to the degeneracy of a wrench system expressed in a matrix form, namely, a $6 \times 6$ Jacobian matrix $\mathbf{J}$. Accordingly, the parallel singularities of a PM correspond to the vanishing conditions of the determinant of $\mathbf{J}$ that can be found using either geometrical [1], symbolic [2] or numerical methods [3].

Lower-mobility PMs are suitable for a wide range of applications that require fewer than six degrees of freedom (dof). The legs of such manipulators apply some constraint wrenches, also known as structural constraints [4], on the end-effector. The wrench system resulting from all the constraints of the legs is called constraint wrench system of the PM, also known as platform constraint system [5]. In turn, the actuators apply some actuation wrenches on

\footnotetext{
*Address all correspondence to this author.
}

the end-effector. The wrench system resulting from all the actuation wrenches of the legs is called actuation wrench system of the PM. In a general configuration, by locking actuators, the end-effector of a PM becomes fully constrained. In screw theory, a zero-pitch wrench corresponds to the Plücker coordinate vector of a finite line in the 3-dimensional projective space $\mathbb{P}^{3}$ whereas an infinite-pitch wrench corresponds to the Plücker coordinate vector of a line at infinity in $\mathbb{P}^{3}$.

For a 6-dof PM, $\mathbf{J}$ is a $6 \times 6$ matrix that transforms the velocities of the actuators to the linear and angular velocities of the end-effector. Zlatanov et al. [6] proposed an approach, using an input-output velocity relationship, to formulate a $6 \times 6$ Jacobian matrix for 6-dof PMs and also for lowermobility PMs whose legs and end-effector have the same $d o f$. Nevertheless, their approach cannot provide a $6 \times 6$ Jacobian matrix for a more general lower-mobility PM and thus it does not allow the examination of all singular configurations of such a PM.

Based on the theory of reciprocal screws [7-11], Joshi and Tsai [12] developed a general methodology to derive a $6 \times 6$ Jacobian matrix $\mathbf{J}$ providing information about both constraint and actuation wrench systems of a lower-mobility PM. Accordingly, the rows of $\mathbf{J}$ for a $(n<6)$-dof PM can be composed of $n$ linearly independent actuation wrenches plus $(6-n)$ linearly independent constraint wrenches. As a result, a lower-mobility PM can exhibit two different types of parallel singularities: (i) constraint singularities [13], also known as platform singularities [5] and (ii) actuation singularities, also called architecture singularities [12]. Constraint singularities correspond to the degeneracy of the constraint wrench system. In such configurations, the end-effector loses some constraints and gains some extra dof. On the other hand, actuation singularities occur if $\mathbf{J}$ is rank deficient while the constraint wrench does not degenerate.

The Grassmann-Cayley Algebra (GCA) was developed by $\mathrm{H}$. Grassmann as a calculus for linear varieties. The $s u$ perbracket of GCA is a determinant of six 2-extensors that are associated with six Plücker lines. Accordingly, by selecting two points on each Plücker line of $\mathbf{J}$, a superbracket expression can be formulated and amounts to the determinant 
of $\mathbf{J}$, up to scale. By exploring the foregoing superbracket thanks to the GCA operators, the singularity conditions of a PM can be obtained. In turn, Grassmann geometry provides a classification of linear varieties.

For Gough-Stewart PMs, the rows of $\mathbf{J}$ are Plücker coordinate vectors of six finite lines that are six actuation forces applied by the actuators on the end-effector. Parallel singularities of such manipulators occur when those lines become linearly dependent, i.e., when the linear variety spanned by these lines degenerates. Merlet [1] analyzed these singularities using Grassmann geometry by using a set of geometric rules for which a linear variety of dimension $n \leq 6$ degenerates. On the other hand, Ben-Horin et al. [14-16] used the superbracket and the GCA operators to analyze the parallel singularities of 6-dof PMs whose legs transmit six pure forces (six finite lines) to the moving platform. In comparison with Grassmann line geometry, GCA makes it possible to work in a coordinate free manner, and therefore, to produce invariant algebraic expressions for the parallel singularities. Accordingly, all the singularity conditions of the manipulator under study can be enumerated.

However, the method proposed in [14-16] cannot be applied when a line at infinity is among the six lines of $\mathbf{J}$, which is the case for lower-mobility PMs with one or several limited rotational dof. Indeed, there are some infinitepitch wrenches (pure moments) among the rows of $\mathbf{J}$ of such manipulators. Since an infinite-pitch wrench corresponds to the Plücker coordinate vectors of a line at infinity, it cannot be represented by finite points in the superbracket. To this end, Kanaan et al. [17] presented a method to formulate a superbracket with some points at infinity and enlarged the application of GCA to lower-mobility PMs.

The main purpose of this paper is to extend the method proposed in [17] to robots with more complex kinematics such as lower-mobility PMs with an articulated nacelle. For such manipulators, the concept of twist graph is introduced in order to simplify their constraint analysis while the wrench graph is obtained in order to represent their wrenches in the projective space. This wrench graph illustrates all the geometric properties between the constraints of the manipulator and allows one to highlight the points at infinity of the superbracket. This approach can be applied to other lowermobility PMs with an articulated nacelle.

This paper is organized as follows: Section 2 recalls some properties of projective space, screw theory and GCA that are useful for the understanding of the paper. Section 3 presents the constraint analysis and the singularity analysis of the $H 4$ robot. Finally, Section 4 enumerates all the singularity conditions of this robot and characterizes the motions associated with these singularities.

\section{Fundamental Concepts}

\subsection{The 3-dimensional projective space}

The 3-dimensional projective space $\mathbb{P}^{3}$ is characterized by the affine space $\mathbb{R}^{3}$ in addition to the plane at infinity $\Omega_{\infty}$. It is noteworthy that the coordinates of a projective element are determined up to scale. A projective point has four homogeneous coordinates whereas a projective line has six Plücker coordinates represented by its Plücker coordinate vector $P_{\mathbf{L}}=\left(P_{4,1}, P_{4,2}, P_{4,3}, P_{2,3}, P_{3,1}, P_{1,2}\right)^{T}$ satisfying the Grassmann Plücker relation $P_{4,1} P_{2,3}+P_{4,2} P_{3,1}+P_{4,3} P_{1,2}=0$. The following properties highlight the relations between projective elements:

1. A finite point, $A$, is represented by its homogeneous coordinates vector $\mathrm{a}=\left(a_{1}, a_{2}, a_{3}, 1\right)^{T}$, the first three coordinates being its Cartesian coordinates in $\mathbb{R}^{3}$;

2. A finite line, $\mathcal{L}$, is represented by its Plücker coordinates vector ${ }^{1} F=(\mathbf{s} ; \mathbf{r} \times \mathbf{s})$; where $\mathbf{s}$ is the unit vector of $\mathcal{L}$, $\mathbf{r}$ is the position vector of any point on $\mathcal{L}$ and $(\mathbf{r} \times \mathbf{s})$ represents the moment of $\mathcal{L}$ with respect to the origin;

3. Let underlined points denote points at infinity. Any finite line, $F=(\mathbf{s} ; \mathbf{r} \times \mathbf{s})$, has a unique point at infinity $\underline{\mathbf{c}}=(\mathbf{s} ; 0)$. This point only depends on the line direction and is determined up to scale. Accordingly, if $\mathrm{a}$ and $\mathrm{b}$ are two finite points on $F$, then $\underline{c}=\mathrm{b}-\mathrm{a}$;

4. All finite lines directed along $\mathbf{S}$ intersect at one common point at infinity, namely, $\underline{\mathrm{c}}$;

5. All finite planes of normal vector $\mathbf{m}$, have a common line at infinity. This line is given by: $M=\left(\mathbf{0}_{3 \times 1} ; \mathbf{m}\right)$ and passes through the point at infinity on any finite line orthogonal to $\mathbf{m}$;

6. Two lines at infinity $M_{1}=\left(\mathbf{0}_{3 \times 1} ; \mathbf{m}_{1}\right)$ and $M_{2}=$ $\left(\mathbf{0}_{3 \times 1} ; \mathbf{m}_{2}\right)$ intersect at a unique point at infinity $\underline{\mathrm{g}}=$ $\left(\mathbf{m}_{1} \times \mathbf{m}_{2} ; 0\right)$.

\subsection{Theory of Reciprocal Screws}

Screw theory is suitable for the type synthesis and the study of the instantaneous motion of PMs. The principle of reciprocal screws was studied in [7-9] and then developed in $[10,13,18]$.

A unit screw is given by $\hat{\$}=\left[\mathbf{s} ;\left(\mathbf{s}_{0} \times \mathbf{s}+\lambda \mathbf{s}\right)\right]$ where $\mathbf{s}$ is a unit vector along the screw axis, $\mathbf{s}_{0}$ is the position vector of a point on the screw axis with respect to a reference frame and $\lambda$ is the pitch of the screw. A screw of intensity $\rho$ could be written as: $\$=\rho \hat{\$}$. A zero-pitch screw $\hat{\$}_{0}=(\mathbf{s} ; \mathbf{r} \times \mathbf{s})$ corresponds to the Plücker coordinate vector of a finite line in $\mathbb{P}^{3}$. An infinite-pitch screw $\hat{\$}_{\infty}=\left(\mathbf{0}_{3 \times 1} ; \mathbf{s}\right)$ corresponds to the Plücker coordinate vector of line at infinity in $\mathbb{P}^{3}$.

A screw system of order $n(0 \leq n \leq 6)$, also called $n$ system, comprises all screws that are linearly dependent on $n$ given linearly independent screws. The reciprocity conditions of finite- and/or infinite-pitch screws are:

(a) Two zero-pitch screws are reciprocal to each other if and only if their axes are coplanar;

(b) A zero-pitch screw $\hat{\$}_{0}$ is reciprocal to an infinite-pitch screw $\hat{\$}_{\infty}$ if their directions are orthogonal to each other;

(c) Two infinite-pitch screws are always reciprocal to each other.

All screws that are reciprocal to a $n$-system of screws $(n<6)$ form a $(6-n)$-system.

\footnotetext{
${ }^{1}$ In the scope of this paper, $(\mathbf{s} ; \mathbf{r} \times \mathbf{s})$ denotes a Plücker coordinate vector given by $\left[\begin{array}{c}\mathbf{s} \\ \mathbf{r} \times \mathbf{s}\end{array}\right]$.
} 
A twist is a screw representing the instantaneous motion of a rigid body, a wrench is a screw representing a system of forces and moments acting on a rigid body. A zero-pitch twist $\hat{\varepsilon}_{0}$ is associated with a pure rotation while an infinitepitch twist $\hat{\varepsilon}_{\infty}$ is associated with a pure translation. A zeropitch wrench $\hat{F}=(\mathbf{s} ; \mathbf{r} \times \mathbf{s})$ represents a pure force whereas an infinite-pitch wrench $\hat{M}=\left(\mathbf{0}_{3 \times 1} ; \mathbf{n}\right)$ represents a pure moment.

In this paper, screw theory is used to determine the Jacobian matrix $\mathbf{J}$ of the manipulator under study. Accordingly, the reciprocity condition is applied in order to characterize, for each leg-chain of the manipulator, the constraint wrench system reciprocal to its twist system as well as the actuation wrench system obtained by locking actuators.

\subsection{Grassmann-Cayley Algebra}

The GCA was developed by H. Grassmann (1809-1877) as a calculus for linear varieties operating on extensors with the join " $\vee$ " and meet " $\wedge$ " operators. The latter are associated with the span and intersection of vector spaces of extensors characterized with their step. In the $(d=4)$-dimensional vector space $V$ associated with $\mathbb{P}^{3}$, extensors of step 1,2 and 3 represent points, lines and planes, respectively. They are also associated with subspaces of $V$ of dimension 1, 2 and 3, respectively. The GCA makes it possible to work at the symbolic level, and therefore, to produce coordinatefree algebraic expressions for the geometric singularity conditions of spatial PMs. In this section, some properties of GCA are recalled. For further details on GCA, the reader is referred to $[15,19-21]$.

\subsubsection{The superbracket decomposition}

Generally, the rows of $\mathbf{J}$ of a PM are Plücker coordinate vectors of six lines in $\mathbb{P}^{3}$. The superjoin of these six vectors in $\mathbb{P}^{5}$ corresponds to the determinant of $\mathbf{J}$ up to a scalar multiple, which is the superbracket in GCA $\Lambda\left(V^{(2)}\right)$ [21]. Thus, a singularity occurs when these six projective lines become linearly dependent, which amounts to a superbracket equal to zero. The superbracket is an expression involving 12 points selected on these lines and can be developed into a linear combination of 24 bracket monomials $y_{i}(i=$ $1, \ldots, 24)[15,22]$, each one being the product of three brackets of four projective points:

$$
[\mathrm{ab}, \mathrm{cd}, \mathrm{ef}, \mathrm{gh}, \mathrm{ij}, \mathrm{kl}]=\sum_{i=1}^{24} y_{i}
$$

These 24 bracket monomials are given in Appendix A. A bracket [abcd] is null if and only if the projective points a, b, c and d are coplanar. The bracket of four projective points is defined as the determinant of the matrix whose columns are the homogeneous coordinates of these points.

\subsubsection{Geometric incidences in $\mathbb{P}^{3}$}

The join and meet operators were used in $[16,17]$ to describe many geometric incidences between elements in the
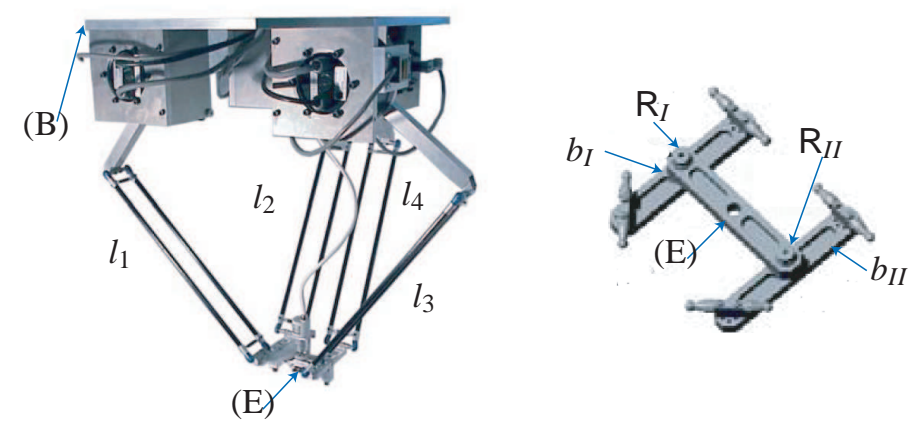

Fig. 1. $H 4$ robot.

projective space $\mathbb{P}^{3}$. For instance, the following geometric incidence developed in Appendix $\mathbf{B}$ is used in Sec. 3.2.

$$
\begin{aligned}
\operatorname{gh} \wedge(\operatorname{abc} \wedge \operatorname{def}) & =[\text { gabc }][\text { hdef }]-[\text { habc }][\text { gdef }] \\
& =[\dot{g} a b c][\text { hdef }]
\end{aligned}
$$

where dotted letters stand for permuted elements as mentioned in [21]. Equation (2) is equal to zero if line (gh) intersects with the intersection line of planes $(a b c)$ and (def).

\section{Singularity Analysis of The $H 4$ Robot}

The $H 4$ robot belongs to a recent family of 4-dof parallel robots designed for high-speed pick and place operations. There exists two varieties of architectures of the $H 4$ family with either revolute or prismatic actuators [23, 24]. The $H 4$ robot shown in Fig. 1 is composed of four identical legs ${ }^{2} l_{i}=\underline{\mathrm{R}}^{i}-(4 \mathrm{~S})^{i}(i=1, \ldots, 4)$ attached to a common base $B$ and linked to the end-effector $E$ by means of an articulated nacelle. The actuated joints are the $\mathrm{R}^{i}$-joints of legs $l_{i}$, $i=1, \ldots, 4$. The axes of $\underline{\mathrm{R}}^{1}$ and $\underline{\mathrm{R}}^{3}$ (respectively $\underline{\mathrm{R}}^{2}$ and $\underline{\mathrm{R}}^{4}$ ) are directed along $\mathbf{y}$ (respectively $\mathbf{x}$ ). The nacelle is composed of three bodies: (i) link $b_{I}$ connecting $l_{1}$ and $l_{2}$ in parallel and making a resulting chain $l_{12}$; (ii) link $b_{I I}$ connecting $l_{3}$ and $l_{4}$ in parallel and making a resulting chain $l_{34}$; (iii) the end-effector $(E)$ linked to $b_{I}$ and $b_{I I}$ with two revolute joints $\mathrm{R}_{I}$ and $\mathrm{R}_{I I}$ having two parallel vertical axes. Finally, the $H 4$ robot has two compound legs: $L_{I}=l_{12}-\mathrm{R}_{I}$ and $L_{I I}=l_{34}-\mathrm{R}_{I I}$.

A $4 \mathrm{~S}$ joint is composed of four links $m n, m p, n q$ and $p q$ connected with four spherical joints centered at $m, n, p$ and $q$, respectively. According to $[23,25]$, the $H 4$ robot is designed in such a way that points $m, n, p$ and $q$ of a $(4 S)^{i}$ joint, represented in Fig 2, form a parallelogram in any robot configuration. In a leg $l_{i}$ shown in Fig. 2 , let $\mathbf{n}_{i}$ denote the unit vector normal to this parallelogram and let $\mathbf{s}_{i}$ and $\mathbf{l}_{i}$ denote the unit vectors along lines $m n$ and $m p$, respectively. Note that lines $m n, p q$ and the axis of the actuated joint $\underline{\mathrm{R}}^{i}$ are parallel. Therefore, $\mathbf{s}_{1}\left\|\mathbf{s}_{3}\right\| \mathbf{y}$ and $\mathbf{s}_{2}\left\|\mathbf{s}_{4}\right\| \mathbf{x}$.

\footnotetext{
${ }^{2} \mathrm{R}, \mathrm{S}$ and (4S) denote a revolute joint, a spherical joint and a spatial
} parallelogram joint, respectively. 


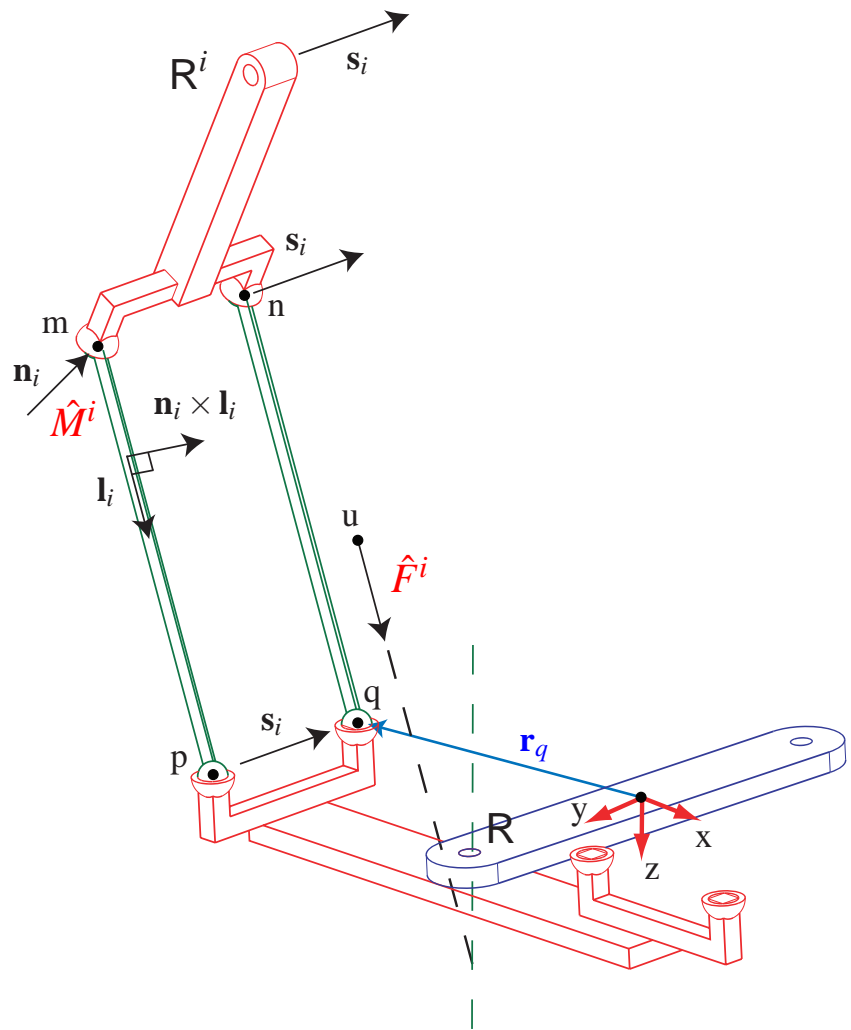

Fig. 2. Leg $l_{i}-\mathrm{R}$ of the $H 4$ robot.

\subsection{Constraint analysis}

Based on the results obtained in $[11,26]$, the twist system of a $(4 S)^{i}$ joint is equivalent to the twist system of a virtual serial chain composed of two virtual revolute joints $R_{1}^{i}$ and $R_{2}^{i}$, a virtual prismatic joint $P^{i}$ and a virtual $\Pi^{i}$-joint ${ }^{3}$ whose associated twists are: $\hat{\varepsilon}_{02}^{i}=\left(\mathbf{s}_{i} ; \mathbf{r}_{n}^{i} \times \mathbf{s}_{i}\right)$, $\hat{\varepsilon}_{03}^{i}=\left(\mathbf{l}_{i} ; \mathbf{r}_{p}^{i} \times \mathbf{l}_{i}\right), \hat{\varepsilon}_{\infty 1}^{i}=\left(\mathbf{0}_{3 \times 1} ; \mathbf{n}_{i}\right)$ and $\hat{\varepsilon}_{\infty 2}^{i}=\left(\mathbf{0}_{3 \times 1} ; \mathbf{l}_{i} \times \mathbf{n}_{i}\right)$, respectively. Accordingly, the twist graph of the $H 4$ robot is shown in Fig. 3. It represents the independent joint twists of the legs. In this graph, lines and circles represent joints and links, respectively whereas dashed lines and dashed circles represent virtual joints and virtual links, respectively. Let $\hat{\varepsilon}_{01}^{i}$ be the twist associated with $\underline{R}^{i}$. The constraint wrench system $\mathrm{W}_{i}$ of leg $l_{i}=\underline{\mathrm{R}}^{i}-(4 \mathrm{~S})^{i}(i=1, \ldots, 4)$ is reciprocal to twists $\hat{\varepsilon}_{01}^{i}, \hat{\varepsilon}_{02}^{i}, \hat{\varepsilon}_{03}^{i}, \hat{\varepsilon}_{\infty 1}^{i}$ and $\hat{\varepsilon}_{\infty 2}^{i}$. Thus, it is a 1 -system spanned by $\hat{M}_{i}$ such that:

$$
\begin{aligned}
& \hat{M}_{1}=\left(\mathbf{0}_{3 \times 1} ; \mathbf{y} \times \mathbf{l}_{1}\right)=\left(\mathbf{0}_{3 \times 1} ; \mathbf{n}_{1}\right) \\
& \hat{M}_{2}=\left(\mathbf{0}_{3 \times 1} ; \mathbf{x} \times \mathbf{l}_{2}\right)=\left(\mathbf{0}_{3 \times 1} ; \mathbf{n}_{2}\right) \\
& \hat{M}_{3}=\left(\mathbf{0}_{3 \times 1} ; \mathbf{y} \times \mathbf{l}_{3}\right)=\left(\mathbf{0}_{3 \times 1} ; \mathbf{n}_{3}\right) \\
& \hat{M}_{4}=\left(\mathbf{0}_{3 \times 1} ; \mathbf{x} \times \mathbf{l}_{4}\right)=\left(\mathbf{0}_{3 \times 1} ; \mathbf{n}_{4}\right)
\end{aligned}
$$

\footnotetext{
${ }^{3} \mathrm{~A} \Pi^{i}$-joint couples two links while allowing a relative translation along a circular trajectory [27].
}

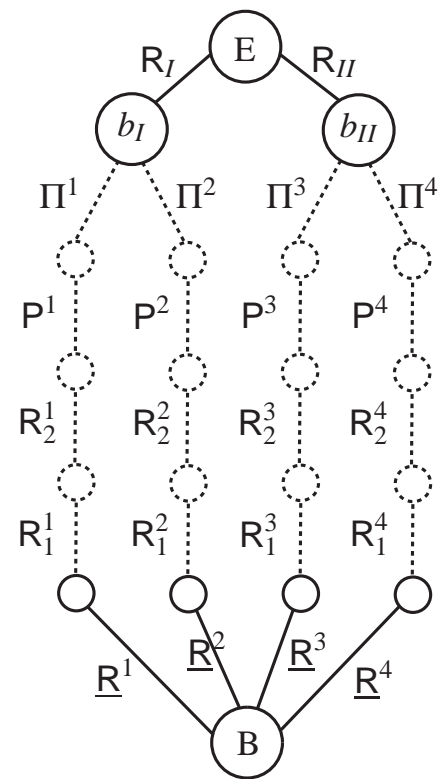

Fig. 3. Twist graph of the $H 4$ robot.

The wrench system $\mathrm{W}_{12}$ and the twist system $\mathrm{T}_{12}$ of chain $l_{12}$ $\operatorname{are}^{4}$ :

$$
\begin{array}{r}
\mathrm{W}_{12}=\mathrm{W}_{1}+\mathrm{W}_{2}=\operatorname{span}\left(\hat{M}_{1}, \hat{M}_{2}\right) \\
\mathrm{T}_{12}=\mathrm{W}_{12}^{\perp}=\operatorname{span}\left(\hat{\varepsilon}_{\infty x}, \hat{\varepsilon}_{\infty y}, \hat{\varepsilon}_{\infty z}, \hat{\varepsilon}_{012}\right)
\end{array}
$$

where $\hat{\varepsilon}_{\infty x}=\left(\mathbf{0}_{3 \times 1} ; \mathbf{x}\right), \hat{\varepsilon}_{\infty y}=\left(\mathbf{0}_{3 \times 1} ; \mathbf{y}\right)$ and $\hat{\varepsilon}_{\infty z}=\left(\mathbf{0}_{3 \times 1} ; \mathbf{z}\right)$ and the axis of $\hat{\varepsilon}_{012}$ is directed along $\mathbf{n}_{12}=\mathbf{n}_{1} \times \mathbf{n}_{2}$. Thus, this chain can be assimilated to a parallel manipulator with three translations and one rotation about an axis directed along $\mathbf{n}_{12}$. Similarly for chain $l_{34}$,

$$
\mathrm{T}_{34}=\mathrm{W}_{34}^{\perp}=\operatorname{span}\left(\hat{\varepsilon}_{\infty x}, \hat{\varepsilon}_{\infty y}, \hat{\varepsilon}_{\infty z}, \hat{\varepsilon}_{034}\right)
$$

where the axis of $\hat{\varepsilon}_{034}$ is directed along $\mathbf{n}_{34}=\mathbf{n}_{3} \times \mathbf{n}_{4}$.

The twist system of leg $L_{I}=l_{12}-\mathrm{R}_{I}$ is $\mathrm{T}_{I}=\mathrm{T}_{12}+\mathrm{T}_{R_{I}}$. $\mathrm{R}_{I}$ is a revolute joint of axis $Z_{I}$ (Fig. 4), represented with the twist $\hat{\varepsilon}_{0 Z_{I}}=\left(\mathbf{z} ; \mathbf{r}_{c} \times \mathbf{z}\right)$. Thus,

$$
\mathrm{T}_{I}=\operatorname{span}\left(\hat{\varepsilon}_{\infty x}, \hat{\varepsilon}_{\infty y}, \hat{\varepsilon}_{\infty z}, \hat{\varepsilon}_{012}, \hat{\varepsilon}_{0 Z_{I}}\right)
$$

Therefore,

$$
\mathrm{W}_{I}=\mathrm{T}_{I}^{\perp}=\operatorname{span}\left(\hat{M}_{I}\right) ; \hat{M}_{I}=\left(\mathbf{0}_{3 \times 1} ; \mathbf{n}_{12} \times \mathbf{z}\right)
$$

Likewise,

$$
\mathrm{W}_{I I}=\operatorname{span}\left(\hat{M}_{I I}\right) ; \hat{M}_{I I}=\left(\mathbf{0}_{3 \times 1} ; \mathbf{n}_{34} \times \mathbf{z}\right)
$$

Finally, the constraint wrench system of the $H 4$ robot is a 2system spanned by two infinite-pitch wrenches of directions

\footnotetext{
${ }^{4}$ The notation " $\perp$ " denotes the reciprocity of two screw systems.
} 

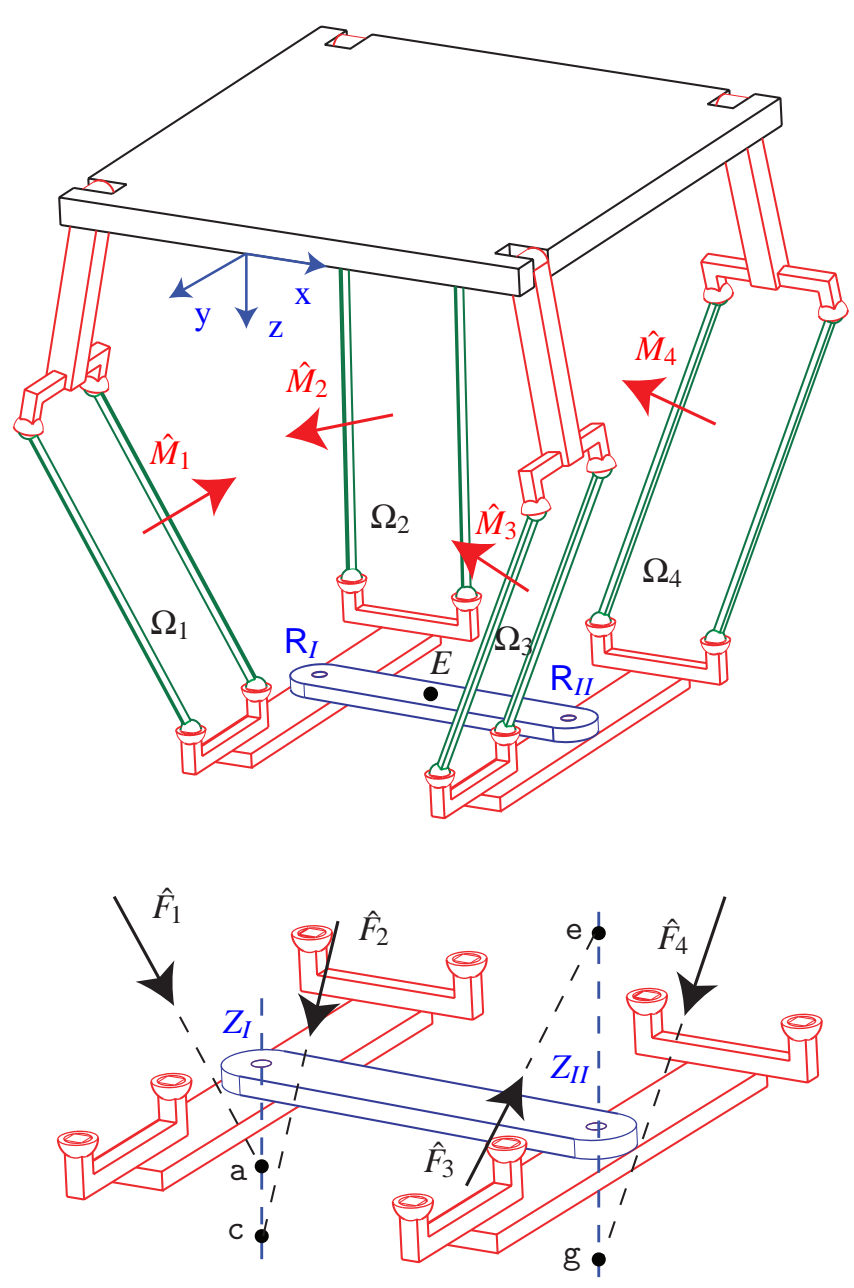

Fig. 4. Constraint and actuation wrenches of the $H 4$ robot.

orthogonal to $\mathbf{z}$, as follows:

$$
\mathrm{W}_{H 4}^{c}=\mathrm{W}_{I}+\mathrm{W}_{I I}=\operatorname{span}\left(\hat{M}_{I}, \hat{M}_{I I}\right)
$$

Accordingly, the end effector $E$ of the robot provides three independent translations and one rotation about an axis of fixed direction along $\mathbf{z}$, namely, a Schönflies motion [28].

Legs $l_{1}$ and $l_{2}$ (respectively $l_{3}$ and $l_{4}$ ) are connected with $\mathrm{R}_{I}$ (respectively $\mathrm{R}_{I I}$ ). The actuated joints of the $H 4$ robot are the $\underline{\mathrm{R}}^{i}$ joints of legs $l_{i}(i=1, \ldots, 4)$. The actuation wrench $\hat{F}_{i}$ of leg $l_{i}, i=1,2$ (respectively $i=3,4$ ), is a zero-pitch wrench of axis parallel to $\mathbf{l}_{i}$, intersecting axis $Z_{I}$ (respectively $Z_{I I}$ ) and lying in the plane $\Omega_{i}$ of the $(4 S)^{i}$ joint. Consequently, $\hat{F}_{i}=\left(\mathbf{l}_{i} ; \mathbf{r}_{u}^{i} \times \mathbf{l}_{i}\right)(i=1, \ldots, 4)$ and the actuation wrench system of the $H 4$ robot is:

$$
\mathrm{W}_{H 4}^{a}=\operatorname{span}\left(\hat{F}_{1}, \hat{F}_{2}, \hat{F}_{3}, \hat{F}_{4}\right)
$$

\subsection{Superbracket of the $H 4$ robot}

The rows of the Jacobian matrix of the $H 4$ robot can be expressed as the six unit screws $\hat{M}_{I}, \hat{M}_{I I}, \hat{F}_{1}, \hat{F}_{2}, \hat{F}_{3}$ and $\hat{F}_{4}$ that correspond to two lines at infinity and four finite lines

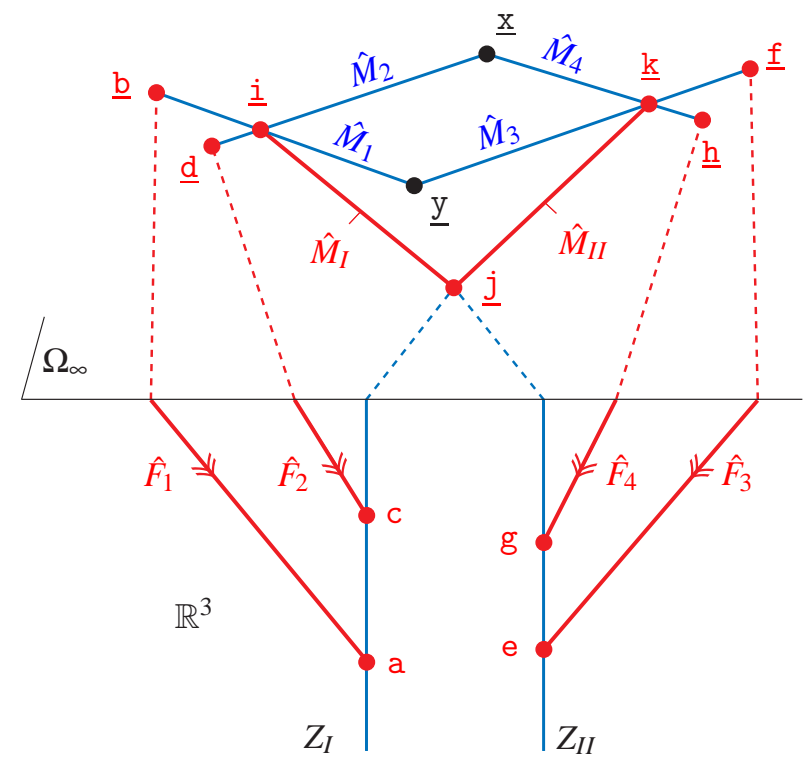

Fig. 5. Wrench graph of the $H 4$ robot in $\mathbb{P}^{3}$.

in $\mathbb{P}^{3}$, respectively. To obtain the twelve points of the superbracket of the robot, we must select two points on each of these six lines. The choice of the latter points must lead to the simplest expression of the superbracket decomposition, i.e., to a reduced number of non-zero monomials. A monomial in Eq. (1) is null if one of its three brackets vanishes. In turn, a bracket is null if its four projective points are coplanar. For example, a bracket taking the following form; [abca] is null.

In order to represent the lines at infinity in the superbracket, it is necessary to use points at infinity. A finite line has one unique point at infinity. Since the set of all points at infinity is a plane $\Omega_{\infty}$, the bracket of four points at infinity is null. Accordingly, in case some lines at infinity are among the six Plücker lines of the $\operatorname{robot}^{5}$, it is convenient to include as many points at infinity as possible in the superbracket. Therefore, a finite line is represented with one finite point and its unique point at infinity.

Since points at infinity cannot be shown in Fig. 4, a representation in $\mathbb{P}^{3}$ of the wrenches of the $H 4$ robot is given in Fig. 5. This representation is based on the properties of the projective space that are given in Sec. 2.

Let a (respectively c) denote the intersection point of $\hat{F}_{1}$ (respectively $\hat{F}_{2}$ ) and $Z_{I}$ and let e (respectively g) denote the intersection point of $\hat{F}_{3}$ (respectively $\hat{F}_{4}$ ) and $Z_{I I}$ (Fig. 4). Besides, $Z_{I}$ and $Z_{I I}$ are directed along $\mathbf{z}$, i.e., ac and eg are parallel finite lines and they intersect in $\Omega_{\infty}$ at $j=(\mathbf{z} ; 0)$, the latter being the point at infinity corresponding to $\mathbf{z}$.

Let $\underline{\mathrm{b}}=\left(\mathbf{l}_{1} ; 0\right), \underline{\mathrm{d}}=\left(\mathbf{l}_{2} ; 0\right), \underline{\mathbf{f}}=\left(\mathbf{l}_{3} ; 0\right)$ and $\underline{\mathrm{h}}=\left(\mathbf{l}_{4} ; 0\right)$ be the points at infinity corresponding to $\mathbf{l}_{1}, \mathbf{l}_{2}, \mathbf{l}_{3}$ and $\mathbf{l}_{4}$, respectively. Therefore,

$$
\hat{F}_{1}=\mathrm{ab} ; \hat{F}_{2}=\mathrm{c} \underline{\mathrm{d}} ; \hat{F}_{3}=\mathrm{e} \underline{\mathrm{f}} ; \hat{F}_{4}=\mathrm{g} \underline{\mathrm{h}}
$$

\footnotetext{
${ }^{5}$ In general, this is the case of lower mobility PMs with at least one limited rotational dof.
} 
The finite line $\hat{M}_{1}=\left(\mathbf{0}_{3 \times 1} ; \mathbf{y} \times \mathbf{l}_{1}\right)$ passes through the points at infinity $\underline{\mathrm{y}}=(\mathbf{y} ; 0)$ and $\underline{\mathrm{b}}=\left(\mathbf{l}_{1} ; 0\right)$;

$$
\hat{M}_{1}=\left(\mathbf{0}_{3 \times 1} ; \mathbf{y} \times \mathbf{l}_{1}\right)=\left(\mathbf{0}_{3 \times 1} ; \mathbf{n}_{1}\right)=\underline{y} \underline{\mathrm{b}}
$$

Likewise,

$$
\hat{M}_{2}=\left(\mathbf{0}_{3 \times 1} ; \mathbf{x} \times \mathbf{l}_{2}\right)=\left(\mathbf{0}_{3 \times 1} ; \mathbf{n}_{2}\right)=\underline{\mathrm{xd}}
$$

Furthermore, $\hat{M}_{1}$ and $\hat{M}_{2}$ intersect at the point at infinity $\underline{\underline{i}}=$ $\left(\mathbf{n}_{12} ; 0\right)$, where $\mathbf{n}_{12}=\mathbf{n}_{1} \times \mathbf{n}_{2}$.

In the same vein,

$$
\begin{aligned}
& \hat{M}_{3}=\left(\mathbf{0}_{3 \times 1} ; \mathbf{y} \times \mathbf{l}_{3}\right)=\left(\mathbf{0}_{3 \times 1} ; \mathbf{n}_{3}\right)=\underline{\mathrm{y}} \underline{\mathbf{f}} \\
& \hat{M}_{4}=\left(\mathbf{0}_{3 \times 1} ; \mathbf{x} \times \mathbf{l}_{4}\right)=\left(\mathbf{0}_{3 \times 1} ; \mathbf{n}_{4}\right)=\underline{\mathrm{xh}}
\end{aligned}
$$

Lines $\hat{M}_{3}$ and $\hat{M}_{4}$ intersect at point $\underline{\mathrm{k}}=\left(\mathbf{n}_{34} ; 0\right)$ where $\mathbf{n}_{34}=$ $\mathbf{n}_{3} \times \mathbf{n}_{4}$. Finally,

$$
\begin{aligned}
& \hat{M}_{I}=\left(\mathbf{0}_{3 \times 1} ; \mathbf{n}_{12} \times \mathbf{z}\right)=\underline{i \underline{j}} \\
& \hat{M}_{I I}=\left(\mathbf{0}_{3 \times 1} ; \mathbf{n}_{34} \times \mathbf{z}\right)=\underline{\mathrm{k}} \underline{j}
\end{aligned}
$$

From Eqs. (15), (20) and (21), the superbracket of the $H 4$ robot takes the following expression:

$$
S_{H 4}=[\mathrm{ab}, \mathrm{c} \underline{\mathrm{d}}, \mathrm{e} \underline{\mathrm{f}}, \mathrm{g} \underline{\mathrm{h}}, \underline{\mathrm{i}} \underline{\mathrm{j}}, \underline{\mathrm{k}} \underline{\mathrm{j}}]
$$

\subsection{Superbracket decomposition}

From Appendix A, $S_{H 4}$ is decomposed into a linear combination of 24 bracket monomials. Since the bracket of four coplanar projective points is null, the superbracket decomposition leads to 5 non-zero monomials as follows:

$$
\begin{aligned}
& S_{H 4}=-[a \underline{b} c \underline{d}][e \underline{f} \underline{h} \underline{j}][g \underline{i} \underline{k} \underline{j}]-[a \underline{b} c \underline{f}][\underline{d} g \underline{h} \underline{j}][e \underline{i} \underline{k} \underline{j}] \\
& +[a \underline{b} \underline{d} \underline{f}][c g \underline{h} \underline{j}][e \underline{i} \underline{k} \underline{j}]+[a \underline{b} c \underline{h}][\underline{d} \text { e } \underline{f} \underline{j}][g \underline{i} \underline{k} \underline{j}] \\
& -[a \underline{b} \underline{d} \underline{h}][c \text { e } \underline{f} \underline{j}][g \underline{i} \underline{k} \underline{j}]
\end{aligned}
$$

We know that

$$
[\mathrm{g} \underline{\mathrm{i}} \underline{\mathrm{k}} \underline{\mathrm{j}}]=\left|\begin{array}{cccc}
g_{1} & i_{1} & k_{1} & j_{1} \\
g_{2} & i_{2} & k_{2} & j_{2} \\
g_{3} & i_{3} & k_{3} & j_{3} \\
1 & 0 & 0 & 0
\end{array}\right|=\left|\begin{array}{ccc}
i_{1} & k_{1} & j_{1} \\
i_{2} & k_{2} & j_{2} \\
i_{3} & k_{3} & j_{3}
\end{array}\right|=[\mathrm{e} \underline{\mathbf{i}} \underline{\mathrm{k}} \underline{\mathrm{j}}]
$$

Hence, $[g \underline{i} \underline{k} j]$ is a common factor of the five monomials of Eq. (23) as follows:

$$
\left.S_{H 4}=[g \underline{i} \underline{k} \underline{j}]\right]\left(\begin{array}{l}
-[a \underline{b} c \underline{d}][e \underline{f} \underline{h} \hat{j}]-[a \underline{b} c \underline{f}][\underline{d} g \underline{h} \hat{j}] \\
+[a \underline{b} \underline{d} \underline{f}][c g \underline{h} \underline{j}]+[a \underline{b} c \underline{h}][\underline{d} \text { e } \underline{f} \underline{j}] \\
-[a \underline{b} \underline{d} \underline{h}][c \text { e } \underline{f} \underline{j}]
\end{array}\right)(25)
$$

From the syzygies or Grassmann-Plücker relations [15,21], it follows that:

$$
\begin{aligned}
{[\mathrm{a} \underline{\mathrm{b}} \mathrm{c} \mathrm{e}][\underline{\mathrm{d}} \underline{\mathrm{f}} \underline{\mathrm{h}} \underline{j}] } & =[\mathrm{a} \underline{\mathrm{b}} \mathrm{c} \underline{\mathrm{d}}][\mathrm{e} \underline{\mathrm{f}} \underline{\mathrm{h}} \underline{\mathrm{j}}]+[\mathrm{a} \underline{\mathrm{b}} \mathrm{c} \underline{\mathrm{f}}][\underline{\mathrm{d}} \mathrm{e} \underline{\mathrm{h}} \underline{\mathrm{j}}] \\
& +[\mathrm{a} \underline{\mathrm{b}} \mathrm{c} \underline{\mathrm{h}}][\underline{\mathrm{d}} \underline{\mathrm{f}} \mathrm{e} \underline{j}]+[\mathrm{a} \underline{\mathrm{b}} \mathrm{c} \underline{j}][\underline{\mathrm{d}} \underline{\mathrm{f}} \underline{\mathrm{h}} \mathrm{e}]
\end{aligned}
$$

We know that: $[\underline{\mathrm{d}} \underline{\mathrm{f}} \underline{\mathrm{h}} \underline{\mathrm{j}}]=0,[\underline{\mathrm{d}} \mathrm{e} \underline{\mathrm{h}} \underline{\mathrm{j}}]=[\underline{\mathrm{d} g} \underline{\mathrm{h}} \underline{\mathrm{j}}]$ and $[\underline{\mathrm{d}} \underline{\mathrm{f}} \mathrm{e}$ j $\underline{\mathrm{j}}]=$ $-[\underline{d} e \underline{f} \underline{j}$. Furthermore, points $a, c$ and $\underline{j}$ being on the same projective line, $[\mathrm{a} \underline{\mathrm{b}} \mathrm{c} \underline{\mathrm{j}}]=0$. Thus,

$$
-[a \underline{b} c \underline{d}][e \underline{f} \underline{h} \underline{j}]-[a \underline{b} c \underline{f}][\underline{d} g \underline{h} \underline{j}]+[a \underline{b} c \underline{h}][\underline{d} e \underline{f} \underline{j}]=0
$$

Therefore,

$$
S_{H 4}=[g \underline{i} \underline{k} \underline{j}]([a \underline{b} \underline{d} \underline{f}][c g \underline{h} \underline{j}]-[a \underline{b} \underline{d} \underline{h}][c \text { e } \underline{f} \underline{j}])
$$

We know that $\underline{j}=g-e$, i.e., $e=g-\underline{j}$. As a result,

$$
[c e \underline{f} \underline{j}]=[c(g-\underline{j}) \underline{f} \underline{j}]=[c g \underline{f} \underline{j}]
$$

Finally,

$$
\begin{aligned}
S_{H 4} & =[\mathrm{g} \underline{i} \underline{\mathrm{k}} \underline{j}]([\mathrm{a} \underline{\mathrm{b}} \underline{\mathrm{d}} \underline{f}][\mathrm{cg} \underline{\mathrm{h}} \underline{j}]-[\mathrm{a} \underline{\mathrm{b}} \underline{\mathrm{d}} \underline{\mathrm{h}}][\mathrm{cg} \underline{\underline{f}} \underline{j}]) \\
& =[\mathrm{g} \underline{\underline{i}} \underline{\mathrm{k}} \underline{j}]([\mathrm{a} \underline{\mathrm{b}} \underline{\mathrm{d}} \underline{\hat{f}}][\mathrm{cg} \underline{\mathrm{h}} \underline{\mathrm{j}}])
\end{aligned}
$$

where dotted letters stand for the permuted elements, as mentioned in [21].

\subsection{Geometric singularity conditions of the $H 4$ robot} From Eq. (29), a parallel singularity occurs when:

1. $[\mathrm{g} \underline{\mathrm{i}} \underline{\mathrm{k}} \underline{\mathrm{j}}]=0 \Leftrightarrow[\underline{\mathrm{i}} \underline{\mathrm{k}} \underline{\mathrm{j}}]=0 \Leftrightarrow \underline{\mathrm{i}}, \underline{\mathrm{j}}$ and $\underline{\mathrm{k}}$ belong to the same projective line. According to Eq. (24) and since $\underline{\underline{i}}=\left(\mathbf{n}_{12} ; 0\right), \underline{\mathbf{k}}=\left(\mathbf{n}_{34} ; 0\right)$ and $\underline{j}=(\mathbf{z} ; 0)$, this condition is expressed in a vector form as follows:

$$
\left(\mathbf{n}_{12} \times \mathbf{n}_{34}\right) \cdot \mathbf{z}=0
$$

where $\mathbf{n}_{12}=\mathbf{n}_{1} \times \mathbf{n}_{2}$ and $\mathbf{n}_{34}=\mathbf{n}_{3} \times \mathbf{n}_{4}$. In turn, $\mathbf{n}_{1}=$ $\mathbf{y} \times \mathbf{l}_{1}, \mathbf{n}_{2}=\mathbf{x} \times \mathbf{l}_{2}, \mathbf{n}_{3}=\mathbf{y} \times \mathbf{l}_{3}$ and $\mathbf{n}_{4}=\mathbf{x} \times \mathbf{l}_{4}$. Thus, Eq. (30) is equivalent to:

$$
\left(\left(\left(\mathbf{y} \times \mathbf{l}_{1}\right) \times\left(\mathbf{x} \times \mathbf{l}_{2}\right)\right) \times\left(\left(\mathbf{y} \times \mathbf{l}_{3}\right) \times\left(\mathbf{x} \times \mathbf{l}_{4}\right)\right)\right) \cdot \mathbf{z}=0
$$

Equation (30) expresses the dependency of the two constraint moments $\hat{M}_{I}=\left(\mathbf{0}_{3 \times 1} ; \mathbf{n}_{12} \times \mathbf{z}\right)=\underline{\underline{i} j}$ and $\hat{M}_{I I}=$ $\left(\mathbf{0}_{3 \times 1} ; \mathbf{n}_{34} \times \mathbf{z}\right)=\underline{\mathrm{k}} \underline{\mathrm{j}}$. Therefore, it corresponds to the constraint singularities in which the robot loses one or two constraints and gains one or two extra dof. 
2. $[\mathrm{a} \underline{\mathrm{b}} \underline{\mathrm{d}} \underline{\dot{f}}][\mathrm{cg} \underline{\mathrm{h}} \underline{\mathrm{j}}]=0 \Leftrightarrow(\mathrm{a} \underline{\mathrm{b}} \underline{\mathrm{d}} \wedge \mathrm{cg} \underline{\mathrm{j}}) \wedge \underline{\mathrm{f}} \underline{\mathrm{h}}=0 \Leftrightarrow$ the projective line $(\underline{f} \underline{h})$ intersects with the intersection line of planes (a $\underline{b} \underline{\mathrm{d}})$ and (c g j $)$ (see Eq. (2)). This condition is expressed in a vector form as follows:

$$
\left(\left(\mathbf{l}_{1} \times \mathbf{l}_{2}\right) \times\left(\left(\mathbf{r}_{g}-\mathbf{r}_{c}\right) \times \mathbf{z}\right)\right) \cdot\left(\mathbf{l}_{3} \times \mathbf{l}_{4}\right)=0
$$

Equation (32) corresponds to the actuation singularities, namely, configurations in which the actuators cannot control the end-effector's motion.

\section{Motions associated with Parallel Singularities}

In a constraint singularity, the motion pattern of a PM may change and its end-effector might gain some limited motion(s). On the other hand, in an actuation singularity, the actuators of a PM cannot control its end effector's motion, namely, the end-effector might generate some infinitesimal motion(s) even when the actuators are locked. In this section, the different cases of both constraint and actuation singularities are enumerated and the gained and/or the uncontrollable motions are interpreted.

\subsection{Constraint singularities and gained motions}

Constraint singularities correspond to the degeneracy of the constraint wrench system, which produces a change in the limited motion of the end-effector. By solving Eq. (30), the conditions for constraint singularities turn out to be:

(a) $\mathbf{n}_{1} \times \mathbf{n}_{2}=\mathbf{0}_{3 \times 1} \Leftrightarrow \mathbf{n}_{1} \| \mathbf{n}_{2}$, i.e., planes $\Omega_{1}$ and $\Omega_{2}$ are parallel and, as a consequence, $\mathbf{n}_{12}=\mathbf{0}_{3 \times 1}$. Since $\mathbf{n}_{1}=$ $\mathbf{y} \times \mathbf{l}_{1}$ and $\mathbf{n}_{2}=\mathbf{x} \times \mathbf{l}_{2}$, this condition can occur if and only if $\mathbf{l}_{1}$ and $\mathbf{l}_{2}$ are parallel to plane $(\mathbf{x} O \mathbf{y})$, i.e., if $\mathbf{n}_{1} \|$ $\mathbf{n}_{2} \| \mathbf{z}$. It means that points $\underline{b}$ and $\underline{\mathrm{d}}$ belong to the line at infinity (xy), which coincides with lines $\hat{M}_{1}$ and $\hat{M}_{2}$. Accordingly, point $\underline{i}$ cannot be defined. In that case, the constraint moment $\hat{M}_{I}$ is null and the robot gains one extra dof. Indeed, the only limited dof is the rotation about an axis directed along $\mathbf{z} \times \mathbf{n}_{34}$;

(b) $\mathbf{n}_{3} \times \mathbf{n}_{4}=\mathbf{0}_{3 \times 1}$ : this condition can be explored similarly to the previous condition;

(c) $\mathbf{n}_{12} \times \mathbf{n}_{34}=\mathbf{0}_{3 \times 1} \Leftrightarrow \mathbf{n}_{12} \| \mathbf{n}_{34}$ : this happens when the intersection line of planes $\Omega_{1}$ and $\Omega_{2}$ becomes parallel to the intersection line of planes $\Omega_{3}$ and $\Omega_{4}$. This implies that $\underline{\underline{i}} \equiv \underline{\mathrm{k}}$. Under this condition, the robot has only one constraint moment $\hat{M}_{I} \equiv \hat{M}_{I I}$ and the end-effector has only one constraint, namely, the rotation about an axis directed along ${ }^{6} \mathbf{z} \times \mathbf{n}_{12} \equiv \mathbf{z} \times \mathbf{n}_{34}$. For example, when $\mathbf{n}_{1} \| \mathbf{n}_{3}$ and $\mathbf{n}_{2} \| \mathbf{n}_{4}$. Such a configuration is illustrated in Fig. 6;

(d) $\mathbf{n}_{12} \| \mathbf{z}$ : this can occur if $\mathbf{n}_{12}=\mathbf{n}_{1} \times \mathbf{n}_{2}$ is parallel to $\mathbf{z}$, i.e., if $\mathbf{n}_{1}=\mathbf{y} \times \mathbf{l}_{1}$ and $\mathbf{n}_{2}=\mathbf{x} \times \mathbf{l}_{2}$ are parallel to plane $(\mathbf{x} O \mathbf{y})$. This implies to have $\mathbf{l}_{1}\|\mathbf{z}\| \mathbf{l}_{2}$, i.e.,

\footnotetext{
${ }^{6}$ Note that all vectors are considered as unit vectors, i.e., $\mathbf{n}_{12} \| \mathbf{n}_{34}$ implies that $\mathbf{n}_{12} \equiv \mathbf{n}_{34}$.
}

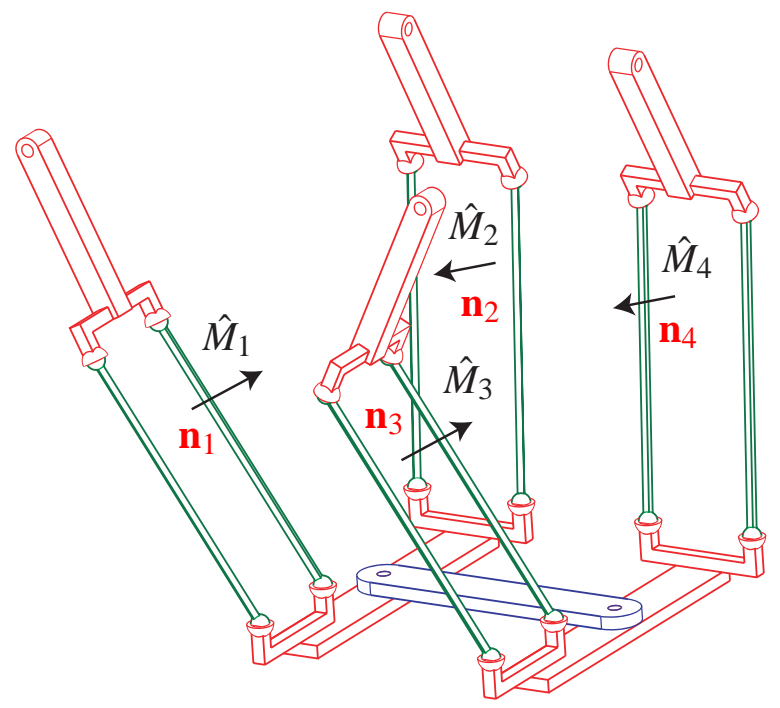

Fig. 6. A constraint singularity: $\left(\mathbf{n}_{1} \times \mathbf{n}_{2}\right) \|\left(\mathbf{n}_{3} \times \mathbf{n}_{4}\right)$.

Table 1. Constraint singularities of the $H 4$ robot.

\begin{tabular}{|c|c|c|c|}
\hline Case & Vector condition & Result & $\operatorname{dim}\left(\mathrm{W}_{H 4}^{c}\right)$ \\
\hline (a) & $\mathbf{n}_{1} \| \mathbf{n}_{2}$ & \multirow[t]{2}{*}{$\hat{M}_{I}$ is null } & \multirow{6}{*}{1} \\
\hline (d) & $\mathbf{n}_{12} \| \mathbf{z}$ & & \\
\hline (b) & $\mathbf{n}_{3} \| \mathbf{n}_{4}$ & \multirow[t]{2}{*}{$\hat{M}_{I I}$ is null } & \\
\hline (e) & $\mathbf{n}_{34} \| \mathbf{z}$ & & \\
\hline (c) & $\mathbf{n}_{12} \| \mathbf{n}_{34}$ & \multirow[t]{2}{*}{$\hat{M}_{I} \equiv \hat{M}_{I I}$} & \\
\hline (g) & $\left(\mathbf{n}_{12} \times \mathbf{n}_{34}\right) \cdot \mathbf{z}=0$ & & \\
\hline (f) & $\mathbf{n}_{12}\left\|\mathbf{n}_{34}\right\| \mathbf{z}$ & $\begin{array}{l}\hat{M}_{I} \text { and } \hat{M}_{I I} \\
\text { are null }\end{array}$ & 0 \\
\hline
\end{tabular}

$\underline{\mathrm{b}} \equiv \underline{\mathrm{j}} \equiv \underline{\mathrm{d}}$. In that case, $\underline{\mathrm{b}} \equiv \underline{\mathrm{d}} \equiv \underline{\mathrm{i}} \equiv \underline{\mathrm{j}}$. Under this condition, the constraint moment $\hat{M}_{I}$ is null and the robot gains one extra $d o f$

(e) $\mathbf{n}_{34} \| \mathbf{z}$ : this condition can be analyzed similarly to the previous condition;

(f) $\mathbf{n}_{12}\left\|\mathbf{n}_{34}\right\| \mathbf{z} \Leftrightarrow \underline{\underline{i}} \equiv \underline{\mathrm{k}} \equiv \underline{\mathrm{j}}$ : in that case, $\hat{M}_{I}$ and $\hat{M}_{I I}$ are null and the robot gains two rotational $d o f$;

(g) $\mathbf{n}_{12}, \mathbf{n}_{34}$ and $\mathbf{z}$ are orthogonal to a same direction but not parallel to each other. In that case: $\hat{M}_{I} \equiv \hat{M}_{I I}$ and the robot gains one rotational $d o f$.

Table 1 illustrates the constraint singularity conditions of the $\mathrm{H} 4$ robot as well as the dimension of the corresponding constraint wrench system $\mathrm{W}_{H 4}^{c}$.

\subsection{Actuation singularities and uncontrolled motions}

Actuation singularities correspond to the rank deficiency of $\mathbf{J}$ while the constraint wrench system does not degenerate. In that case, the wrenches $\hat{F}_{1}, \hat{F}_{2}, \hat{F}_{3}, \hat{F}_{4}, \hat{M}_{1}$ and $\hat{M}_{2}$ form a $n<6$-system and the twists reciprocal to this system for a given actuation singularity determine the uncontrolled mo- 


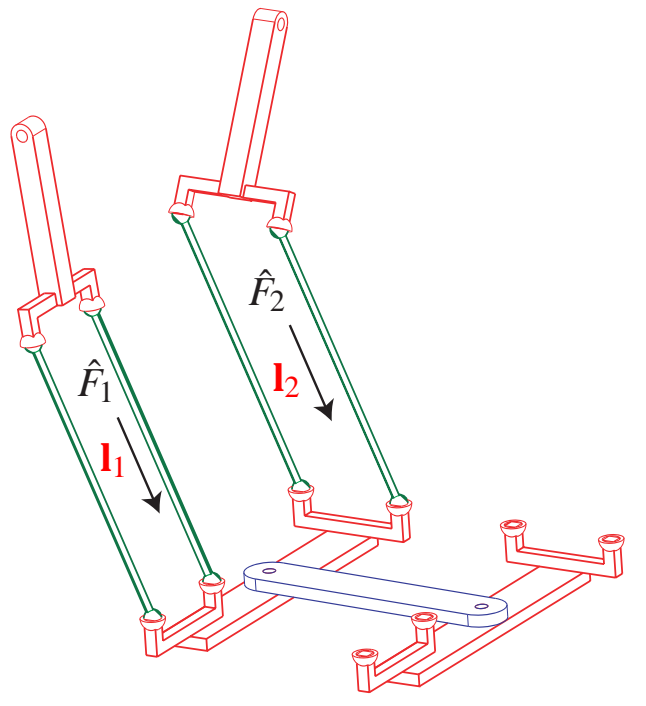

Fig. 7. An actuation singularity: $\mathbf{l}_{1} \| \mathbf{l}_{2}$.

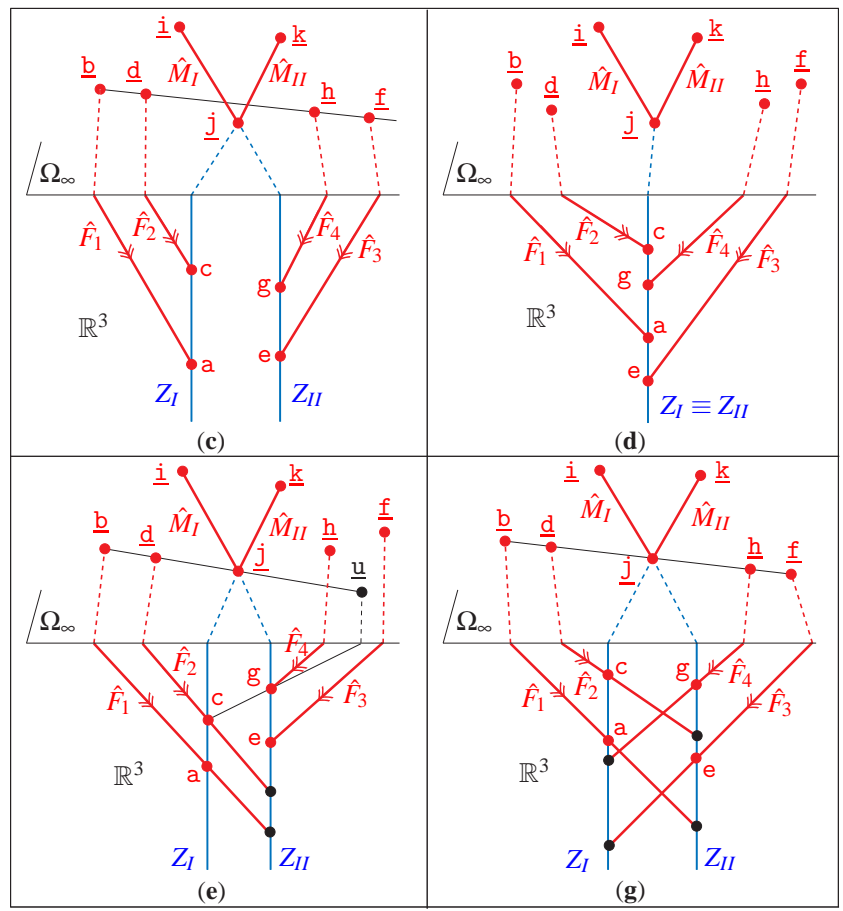

Fig. 8. Wrench graph associated with the actuation singularity conditions $(\mathbf{c}),(\mathbf{d}),(\mathbf{e})$ and $(\mathbf{g})$.

tions of the end-effector [29]. From Eq. (32), an actuation singularity occurs in the following cases:

(a) $\mathbf{l}_{1} \times \mathbf{l}_{2}=0 \Leftrightarrow \mathbf{l}_{1} \| \mathbf{l}_{2} \Leftrightarrow \underline{\mathrm{b}} \equiv \underline{\mathrm{d}}$ : plane (a $\underline{\mathrm{b}} \underline{\mathrm{d}}$ ) degenerates into a line. In that case, the actuation forces $\hat{F}_{1}$ and $\hat{F}_{2}$ are parallel as illustrated in Fig. 7. By applying a linear transformation [26] the wrench system $\operatorname{span}\left(\hat{F}_{1}, \hat{F}_{2}\right)$ is equivalent to $\operatorname{span}\left(\hat{F}_{1}, \hat{M}_{I}\right)$. Consequently, the wrench system $\operatorname{span}\left(\hat{F}_{1}, \hat{F}_{2}, \hat{M}_{I}\right)$ degenerates. However, in that case, one can find neither a zero-pitch twist nor an infinite-pitch twist reciprocal to the six wrenches of $\mathbf{J}$. As a result, in such a configuration, the uncontrolled
Table 2. Actuation singularities of the $H 4$ robot.

\begin{tabular}{l|l}
\hline \hline Case(s) & Uncontrolled motions \\
\hline$(\mathbf{a}),(\mathbf{b}),(\mathbf{h})$ & A combined 1-dof motion \\
\hline$(\mathbf{c})$ & A translation along $\mathbf{l}_{1} \times \mathbf{l}_{2}$ \\
\hline$(\mathbf{d}),(\mathbf{e}),(\mathbf{f})$ & A rotation about an axis directed along $\mathbf{z}$ \\
\hline$(\mathrm{g})$ & A translation along $\mathbf{l}_{1} \times \mathbf{l}_{2}$ plus \\
& a rotation about an axis directed along $\mathbf{z}$ \\
\hline \hline
\end{tabular}

motion of the end-effector is a finite-pitch twist, i.e., a combination of a translation and a rotation;

(b) $\mathbf{l}_{3} \times \mathbf{l}_{4}=0 \Leftrightarrow \mathbf{I}_{3} \| \mathbf{l}_{4} \Leftrightarrow \underline{\mathbf{f}} \equiv \underline{\mathrm{h}}$ : line $(\underline{\mathbf{f}} \underline{\mathrm{h}})$ degenerates into a point. This case can be analyzed similarly to the previous one;

(c) $\left(\mathbf{l}_{1} \times \mathbf{l}_{2}\right) \|\left(\mathbf{l}_{3} \times \mathbf{l}_{4}\right)$ : lines $(\underline{b} \underline{d})$ and $(\underline{f} \underline{h})$ coincide and points $\underline{b}, \underline{d}, \underline{f}$ and $\underline{\mathrm{h}}$ are aligned as shown in Fig. 8(c). In that case, the line at infinity $(\underline{b} \underline{\mathrm{d}}) \equiv(\underline{\mathrm{f}} \underline{\mathrm{h}})$ crosses all the wrenches of $\mathbf{J}$. In other words, one can find an infinite pitch twist $\left(\mathbf{0}_{3 \times 1} ; \mathbf{l}_{1} \times \mathbf{l}_{2}\right)$ reciprocal to all the wrenches of $\mathbf{J}$. Consequently, the actuators cannot control the translational dof of the end-effector along $\mathbf{l}_{1} \times \mathbf{l}_{2}$;

(d) $\left(\mathbf{r}_{g}-\mathbf{r}_{c}\right) \times \mathbf{z}=0$ : in that case, line (cg) is parallel to $\mathbf{z}$. It means that axes $Z_{I}$ and $Z_{I I}$ coincide. Thus, axis $Z_{I} \equiv$ $Z_{I I}$ crosses all the wrenches of $\mathbf{J}$ as shown in Fig. 8(d). As a result, in such a configuration, the actuators cannot control the rotational dof of the end-effector about an axis directed along $\mathbf{z}$;

(e) $\left(\mathbf{l}_{1} \times \mathbf{l}_{2}\right)$ is parallel to $\left(\mathbf{r}_{g}-\mathbf{r}_{c}\right) \times \mathbf{z}$. In that case, points $\underline{\mathrm{b}}, \underline{\mathrm{d}}, \underline{\mathrm{j}}$ and $\underline{\mathrm{u}}=(\mathbf{u} ; 0)$ are aligned and $\mathbf{l}_{1}, \mathbf{l}_{2}, \mathbf{z}$ and $\mathbf{u}=$ $\left(\mathbf{r}_{g}-\overline{\mathbf{r}}_{c}\right)$ are orthogonal to a given direction. Figure 8(e) illustrates the wrench graph of the $H 4$ robot for such a singularity. Thus, the actuation forces $\hat{F}_{1}$ and $\hat{F}_{2}$ lie in plane (cg j $)$. Consequently, in such a configuration, one can find one finite line, namely, $\left(\mathbf{z} ; \mathbf{r}_{g} \times \mathbf{z}\right)$ that crosses all the wrenches of $\mathbf{J}$. Accordingly, the uncontrolled motion of end-effector is a pure rotation about an axis directed along $\mathbf{z}$;

(f) $\left(\mathbf{l}_{3} \times \mathbf{l}_{4}\right)$ is parallel to $\left(\mathbf{r}_{g}-\mathbf{r}_{c}\right) \times \mathbf{z}$. This case is similar to the previous one;

(g) The three vectors $\left(\mathbf{l}_{1} \times \mathbf{l}_{2}\right),\left(\mathbf{l}_{3} \times \mathbf{l}_{4}\right)$ and $\left(\mathbf{r}_{g}-\mathbf{r}_{c}\right) \times \mathbf{z}$ are parallel to each other. In that case, points $\underline{b}, \underline{d}, \underline{f}, \underline{h}, \underline{j}$ and $\underline{\mathbf{u}}=(\mathbf{u} ; 0)$ are aligned and $\mathbf{l}_{1}, \mathbf{l}_{2}, \mathbf{l}_{3}, \mathbf{l}_{4}, \mathbf{z}$ and $\mathbf{u}=\left(\mathbf{r}_{g}-\mathbf{r}_{c}\right)$ are orthogonal to a given direction. Thus, the actuation forces $\hat{F}_{1}, \hat{F}_{2}, \hat{F}_{3}$ and $\hat{F}_{4}$ lie in plane $(\mathrm{cg} \underline{j})$ as shown in Fig. 8(g). Consequently, in such a configuration, one can find two projective lines $\left(\mathbf{z} ; \mathbf{r}_{g} \times \mathbf{z}\right)$ and $\left(\mathbf{0}_{3 \times 1} ; \mathbf{l}_{1} \times \mathbf{l}_{2}\right)$ that cross all the wrenches of $\mathbf{J}$. Accordingly, the endeffector has two uncontrolled dof: a translation along $\mathbf{l}_{1} \times \mathbf{l}_{2}$ and a rotation about an axis directed along $\mathbf{z}$;

(h) The three vectors $\left(\mathbf{l}_{1} \times \mathbf{l}_{2}\right),\left(\mathbf{l}_{3} \times \mathbf{l}_{4}\right)$ and $\left(\mathbf{r}_{g}-\mathbf{r}_{c}\right) \times \mathbf{z}$ are orthogonal to a same direction but not parallel to each other. In such a configuration, the uncontrolled motion of the end-effector corresponds to a finite-pitch twist. 
Table 2 illustrates the uncontrolled motions of the $H 4$ robot associated with the different actuation singularities.

\section{Discussion}

The main contributions of this paper is to apply the GCA for the singularity analysis of lower-mobility PMs with complex kinematics as well as the determination of the motions associated with the parallel singular configurations of such PMs. In comparison with other singularity analysis methods, the GCA provides, through the superbracket decomposition, an enumeration of all the singularity conditions. The singularity analysis of the $H 4$ robot was performed in $[30,31]$ where the condition for constraint singularities expressed in Eq. (30) was obtained. However, the condition for actuation singularities had not been obtained in a complete and general form before, such as the one given in Eq. (32). The results obtained with GCA for the singularity analysis of the $H 4$ robot are consequently more general than the results obtained previously. Moreover, this paper shows that with GCA, the interpretation of singular configurations can be performed based on either a vector condition or an invariant algebraic expression between some projective points of the wrench graph.

On the other side, the constraint and singularity analysis of the $H 4$ robot in $[11,32]$ focused more on screw theory. Here, more emphasis is given on GCA, the selection of the superbracket expression and the simplification of the superbracket decomposition. Indeed, the singularity analysis is completed and all the singularity conditions are enumerated. Furthermore, the motions associated with a given singular configuration of the $H 4$ robot are determined for the first time in this paper.

\section{Conclusions}

This paper presented a systematic approach based on Grassmann-Cayley Algebra (GCA) to analyze the singularities of Lower-Mobility PMs with an articulated nacelle and to characterize the motion associated with a given singular configuration. The proposed approach can be considered as an extension of the method proposed in [17] to manipulators with an articulated nacelle. It was explained through the singularity analysis of the $H 4$ robot. The results provided two geometric conditions that make it possible to enumerate and interpret all the singular configurations of the $H 4$ robot.

The proposed approach consists of three main steps. First, the twist graph of the manipulator is obtained. This twist graph represents the independent twists between the base and the end-effector and is used in order to simplify the constraint analysis of the manipulator. Then, the wrench graph of the manipulator is obtained. This wrench graph is very useful for the singularity analysis of robots with an articulated nacelle. It represents the wrenches, obtained with the constraint analysis, in the projective space $\mathbb{P}^{3}$. Moreover, it allows one to select and express the points at infinity of the superbracket. Finally, the superbracket is analyzed in order to determine and interpret the parallel singularity conditions of the manipulator. These three steps can be followed to perform an exhaustive determination of the singularity conditions of lower-mobility PMs, mainly those having an articulated nacelle like the Par4 ${ }^{7}$ robot [33].

\section{Appendices \\ Appendix A}

The 24 monomials of Eq. (1) are expressed below:

$$
\begin{aligned}
& y_{1}=-[\mathrm{abcd}][\mathrm{efgi}][\mathrm{hjkl}] \quad y_{2}=[\mathrm{abcd}][\mathrm{efhi}][\mathrm{gjkl}] \\
& y_{3}=[\mathrm{abcd}][\mathrm{efgj}][\mathrm{hikl}] \quad y_{4}=-[\mathrm{abcd}][\mathrm{efhj}][\mathrm{gikl}] \\
& y_{5}=[\text { abce }][\mathrm{dfgh}][\mathrm{ijkl}] \quad y_{6}=-[\text { abde }][\mathrm{cfgh}][\mathrm{ijkl}] \\
& y_{7}=-[\mathrm{abcf}][\operatorname{degh}][i j \mathrm{kl}] \quad y_{8}=[\mathrm{abdf}][\mathrm{cegh}][i j \mathrm{kl}] \\
& y_{9}=-[\mathrm{abce}][\mathrm{dghi}][\mathrm{fjkl}] \quad y_{10}=[\mathrm{abde}][\mathrm{cghi}][\mathrm{fjkl}] \\
& y_{11}=[\mathrm{abcf}][\mathrm{dghi}][\mathrm{ejkl}] \quad y_{12}=[\mathrm{abce}][\mathrm{dghj}][\mathrm{fikl}] \\
& y_{13}=-[\operatorname{abdf}][\operatorname{cghi}][\mathrm{ejkl}] \quad y_{14}=-[\operatorname{abde}][\mathrm{cghj}][\mathrm{fikl}] \\
& y_{15}=-[\mathrm{abcf}][\mathrm{dghj}][\mathrm{eikl}] \quad y_{16}=[\mathrm{abdf}][\mathrm{cghj}][\mathrm{eikl}] \\
& y_{17}=[\mathrm{abcg}][\operatorname{def} \mathrm{i}][\mathrm{hjkl}] \quad y_{18}=-[\mathrm{abdg}][\operatorname{cef} \mathrm{i}][\mathrm{hjkl}] \\
& y_{19}=-[\mathrm{abch}][\operatorname{def} \mathrm{i}][\mathrm{gjkl}] \quad y_{20}=-[\mathrm{abcg}][\operatorname{def} j][\mathrm{hikl}] \\
& y_{21}=[\mathrm{abdh}][\operatorname{cefi}][\mathrm{gjkl}] \quad y_{22}=[\mathrm{abdg}][\operatorname{cef} j][\mathrm{hikl}] \\
& y_{23}=[\mathrm{abch}][\operatorname{def} j][\mathrm{gikl}] \quad y_{24}=-[\mathrm{abdh}][\operatorname{cef} j][\mathrm{gikl}]
\end{aligned}
$$

\section{Appendix B}

Let $(\mathrm{abc})$ and (def) be two extensors in $V(d=4)$ of steps $i$ and $j,(i=j=3)$ representing two distinct planes in $\mathbb{P}^{3}$ and let (gh) be an extensor of step $k=2$ (a 2-extensor) representing a line in $\mathbb{P}^{3}$ such that $(\mathrm{gh}) \notin(\mathrm{abc})$ and $(\mathrm{gh}) \notin$ (def). Now let us calculate a bracket expression of $\mathrm{gh} \wedge$ $(a b c \wedge d e f)$. Since the meet operator is associative, one has:

$$
\operatorname{gh} \wedge(\mathrm{abc} \wedge \operatorname{def})=(\mathrm{gh} \wedge \mathrm{abc}) \wedge \operatorname{def}
$$

Since $k+i>d$, the meet $(\mathrm{gh} \wedge \mathrm{abc})$ is an extensor of step $k+i-d=1$ representing the intersection point of line (gh) and plane $(\mathrm{abc})$ expressed as:

$$
\mathrm{gh} \wedge \mathrm{abc}=[\mathrm{gabc}] \mathrm{h}-[\mathrm{habc}] \mathrm{g}
$$

Then $(\mathrm{gh} \wedge \mathrm{abc}) \wedge \operatorname{def}$ is an extensor of step $1+j-d=0$, namely, a scalar that takes the form:

$$
\operatorname{gh} \wedge \mathrm{abc} \wedge \operatorname{def}=[\mathrm{gabc}][\text { hdef }]-[\text { habc }][\text { gdef }]
$$

\section{References}

[1] Merlet, J. P., 1989. "Singular Configurations of Parallel Manipulators and Grassmann Geometry". The International Journal of Robotics Research, 8(5), pp. 45-56.

[2] Angeles, J., Caro, S., and and A. Morozov, W. K., 2006. "The Design and Prototyping of an Innovative Schonflies Motion Generator". IMechE Part C, Journal of

\footnotetext{
${ }^{7}$ The commercial name of the Par4 robot is Quattro (http://www.adept.com/).
} 
Mechanical Engineering Science, special issue: Kinematics, Kinematic Geometry and their applications, 220(C7), July, pp. 935-944.

[3] Merlet, J. P., 2007. "A Formal-Numerical Approach for Robust In-Workspace Singularity Detection”. IEEE Transactions on Robotics, 23(3), June, pp. 393-402.

[4] Li, Q., and Huang, Z., 2003. "Mobility analysis of Lower-mobility Parallel Manipulators Based on Screw Theory". In IEEE International Conference on Robotics and Automation, pp. 1179-1184.

[5] Fang, Y., and Tsai, L. W., 2002. "Structure Synthesis of a Class of 4-DoF and 5-DoF Parallel Manipulators with Identical Limb Structures". The International Journal of Robotics Research, 21(9), pp. 799-810.

[6] Zlatanov, D., Fenton, R. G., and Benhabib, B., 1994. "Singularity Analysis of Mechanisms and Robots Via a Velocity-Equation Model of the Instantaneous Kinematics". In IEEE International Conference on Robotics and Automation, pp. 986-991.

[7] Ball., R. S., 1900. A Treatise On the Theory of Screws. Cambridge University Press, Cambridge, CA.

[8] Waldron, K. J., 1969. "The Mobility of Linkages". PhD Thesis, Stanford University, Cambridge, CA.

[9] Hunt, K. H., 1978. Kinematic Geometry of Mechanisms. Clarendon Press, Oxford.

[10] Kong, X., and Gosselin, C., 2007. Type Synthesis of Parallel Mechanisms, Vol. 33. Springer, Heidelberg.

[11] Amine, S., Kanaan, D., Caro, S., and Wenger, P., 2010. "Constraint and Singularity Analysis of LowerMobility Parallel Manipulators with Parallelogram Joints". In ASME 2010 International Design Engineering Technical Conferences, no. 28483 in DETC2010.

[12] Joshi, S. A., and Tsai, L. W., 2002. "Jacobian Analysis of Limited-DOF Parallel Manipulators". ASME Journal of Mechanical Design, 124(2), June, pp. 254-258.

[13] Zlatanov, D., Bonev, I., and Gosselin, C. M., 2002. "Constraint Singularities of Parallel Mechanisms". In IEEE International Conference on Robotics and $\mathrm{Au}-$ tomation, pp. 496-502.

[14] Ben-Horin, P., and Shoham, M., 2005. "Singularity Analysis of Parallel Robots Based on GrassmannCayley Algebra". In International Workshop on Computational Kinematics.

[15] Ben-Horin, P., and Shoham, M., 2006. "Singularity Analysis of a Class of Parallel Robots Based on Grassmann-Cayley Algebra". Mechanism and Machine Theory, 41(8), pp. 958-970.

[16] Ben-Horin, P., and Shoham, M., 2009. "Application of Grassmann-Cayley Algebra to Geometrical Interpretation of Parallel Robot Singularities". The International Journal of Robotics Research, 28(1), pp. 127-141.

[17] Kanaan, D., Wenger, P., Caro, S., and Chablat, D., 2009. "Singularity Analysis of Lower-Mobility Parallel Manipulators using Grassmann-Cayley Algebra". IEEE Transactions on Robotics, 25, pp. 995-1004.

[18] Mohamed, M., and Duffy, J., 1985. "A Direct Determination of The Instantaneous Kinematics of Fully Parallel Robot Manipulators". ASME Journal of
Mechanisms, Transmissions and Automation in Design, 107(2), pp. 226-229.

[19] White, N. L., 1975. "The Bracket Ring of a Combinatorial Geometry. I'. Transactions of the American Mathematical Society, 202, pp. 79-95.

[20] White, N. L., 1983. "The Bracket of 2-Extensors". Congressus Numerantium, 40, pp. 419-428.

[21] White, N. L., 2005. Grassmann-Cayley Algebra and Robotics Applications, Vol. VIII. Handbook of Geometric Computing.

[22] McMillan, T., 1990. "Invariants of Antisymmetric Tensors". PhD Thesis, University of Florida.

[23] Pierrot, F., and Company, O., 1999. "H4: A New Family of 4-dof Parallel Robots". In IEEE / ASME Int. Conf. on Advanced Intelligent Mechatronics, pp. 508513.

[24] Pierrot, F., Marquet, F., Company, O., and Gil, T., 2001. "H4 Parallel Robot: Modeling, Design and Preliminary Experiments". In IEEE International Conference on Robotics and Automation, Vol. 4, pp. 3256-3261.

[25] Gregorio, R. D., 2004. "Determination of Singularities in Delta-Like Manipulators". The International Journal of Robotics Research, 23(1), January, pp. 89-96.

[26] Zhao, T. S., Dai, J. S., and Huang, Z., 2002. "Geometric Analysis of Overconstrained Parallel Manipulators with Three and Four Degrees of Freedom". JSME International Journal Ser C Mechanical Systems, Machine Elements Manufacturing, 45(3), pp. 730-740.

[27] Angeles, J., 2004. "The Qualitative Synthesis of Parallel Manipulators". ASME Journal of Mechanical Design, 126(4), July, pp. 617-624.

[28] Caro, S., Khan, W. A., Pasini, D., and Angeles, J., 2010. "The Rule-based Conceptual Design of the Architecture of Serial Schönflies-motion Generators". Mechanism and Machine Theory, 45(2), pp. 251-260.

[29] Wolf, A., and Shoham, M., 2003. "Investigation of Parallel Manipulators using Linear Complex Approximation". ASME Journal of Mechanical Design, 125, pp. 564-572.

[30] Krut, S., 2003. “Contribution l'étude des robots parallèles légers, 3T-1R et 3T-2R, à forts débattements angulaires". PhD thesis, Université Montpellier II.

[31] Wu, J., Yin, Z., and Xiong, Y., 2006. "Singularity Analysis of a Novel 4-dof Parallel Manipulator H4". The International Journal of Advanced Manufacturing Technology, 29, pp. 794-802.

[32] Amine, S., Kanaan, D., Caro, S., and Wenger, P., 2010. "Singularity Analysis of Lower-Mobility Parallel Robots with an Articulated Nacelle". In Advances in Robot Kinematics: Motion in Man and Machine 2010, Part 5, Springer, pp. 273-282.

[33] Nabat, V., de la O Rodriguez, M., Company, O., Krut, S., and Pierrot, F., 2005. "Par4: Very High Speed Parallel Robot for Pick-and-Place". In Intelligent Robots and Systems, IEEE/RSJ International Conference, pp. 553558. http://www.adept.com/. 